\title{
Orthostatic hypotension following spinal cord injury: understanding clinical pathophysiology
}

\author{
VE Claydon*,1, JD Steeves ${ }^{1}$ and A Krassioukov ${ }^{1,2,3,4}$ \\ ${ }^{1}$ International Collaboration On Repair Discoveries (ICORD), University of British Columbia, Vancouver, BC, \\ Canada; ${ }^{2}$ Division of Physical Medicine and Rehabilitation, University of British Columbia, Vancouver, BC, Canada, \\ ${ }^{3}$ School of Rehabilitation, University of British Columbia, Vancouver, BC, Canada; ${ }^{4}$ Department of Medicine, \\ University of British Columbia, Vancouver, BC, Canada
}

\begin{abstract}
Motor and sensory deficits are well-known consequences of spinal cord injury (SCI). During the last decade, a significant number of experimental and clinical studies have focused on the investigation of autonomic dysfunction and cardiovascular control following SCI. Numerous clinical reports have suggested that unstable blood pressure control in individuals with SCI could be responsible for their increased cardiovascular mortality. The aim of this review is to outline the incidence and pathophysiological mechanisms underlying the orthostatic hypotension that commonly occurs following SCI. We describe the clinical abnormalities of blood pressure control following SCI, with particular emphasis upon orthostatic hypotension. Possible mechanisms underlying orthostatic hypotension in SCI, such as changes in sympathetic activity, altered baroreflex function, the lack of skeletal muscle pumping activity, cardiovascular deconditioning and altered salt and water balance will be discussed. Possible alterations in cerebral autoregulation following SCI, and the impact of these changes upon cerebral perfusion are also examined. Finally, the management of orthostatic hypotension will be considered. Spinal Cord (2006) 44, 341-351. doi:10.1038/sj.sc.3101855; published online 22 November 2005
\end{abstract}

Keywords: orthostatic hypotension; spinal cord injury

\section{Introduction}

Spinal cord injury (SCI) is a devastating medical condition affecting up to 52.5 individuals per million people in North America alone. ${ }^{1}$ In addition to the motor and sensory deficits associated with SCI, coincident autonomic nervous system impairments are common. On a daily basis, individuals with SCI face the challenge of managing their unstable blood pressure, which frequently results in persistent hypotension and/or episodes of uncontrolled hypertension. Blood pressure control depends upon tonic activation of spinal sympathetic preganglionic neurons (SPN) by descending input from the supraspinal structures. ${ }^{2}$ Following SCI, these pathways are disrupted, and spinal circuits become solely responsible for the generation of sympathetic activity. ${ }^{3}$ This results in a variety of cardiovascular abnormalities that have been well documented in human studies, as well as in animal models. ${ }^{4-8}$ However, the recognition and management of cardiovascular dysfunctions following SCI represent challenging clinical issues.

*Correspondence: VE Claydon, International Collaboration On Repair Discoveries (ICORD), 6270 University Boulevard, University of British Columbia, Vancouver, BC, Canada V6T 1Z4
Moreover, cardiovascular disorders in the acute and chronic stages of SCI are among the most common causes of death in individuals with SCI. ${ }^{9-11}$

This review will present a summary of the current information on the autonomic control of blood pressure in SCI individuals, with particular emphasis on orthostatic hypotension. Orthostatic hypotension represents a complicated clinical condition, which can severely affect quality of life, and is typically difficult to manage. This review will focus on the prevalence of orthostatic hypotension in SCI patients, and the possible mechanisms underlying this disorder.

\section{Physiology and neuroanatomy of cardiovascular control}

When considering pathophysiology, it is always beneficial to first examine the normal physiological state (Figure 1). In able-bodied individuals, both heart rate and blood pressure are controlled by coordinated inputs from two components of the autonomic nervous system: the sympathetic and parasympathetic. These two systems generally have opposing roles and are 


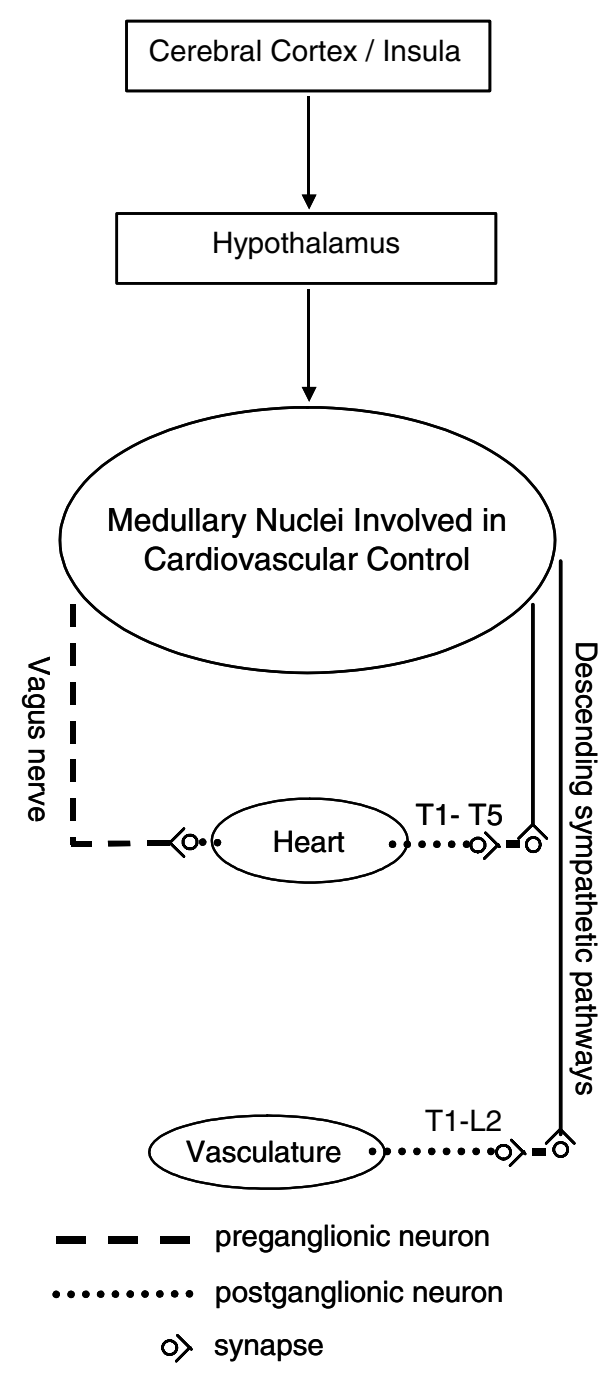

Figure 1 Schematic diagram of autonomic cardiovascular control. The cerebral cortex and hypothalamus project to the various nuclei within the medulla oblongata, where cardiovascular autonomic control is coordinated. The parasympathetic control of the cardiovascular system exits at the level of the brainstem via the vagus nerve. The preganglionic fibres of the vagus nerve then synapse with postganglionic parasympathetic neurons in ganglia on or near the target organ. Descending sympathetic pathways provide tonic control to SPN involved in cardiovascular control. SPN are found within the lateral horn of the spinal cord in segments T1-L2 and exit the spinal cord via the ventral root. They then synapse with postganglionic neurones located in the sympathetic chain (paravertebaral ganglia). Finally, the sympathetic postganglionic neurones synapse with the target organs, the heart and blood vessels

activated according to the needs of the individual. The parasympathetic nervous system is dominant during rest. When stimulated, this system acts to decrease heart rate. The sympathetic nervous system, on the other hand, is largely excitatory, and can be simplistically considered as that which prepares the body for an emergency, forming part of the well-known 'fight and flight reaction', and typically counteracts the parasympathetic nervous system. When the sympathetic nervous system is activated it increases heart rate and the force of myocardial contractions, and increases peripheral vascular resistance, resulting in an increase in blood pressure.

In able-bodied individuals, certain cortical structures, particularly the insula and the hypothalamus, contribute to the autonomic regulation of cardiovascular control. ${ }^{12}$ However, medullary neurons localised within the rostral ventrolateral medullar (RVLM) are thought to be responsible for the maintenance of peripheral vascular tone and arterial blood pressure. ${ }^{2,13}$ RVLM neurons normally provide tonic innervation to the SPN located within the lateral horns of the spinal grey matter of the thoracic and upper lumbar spinal segments. ${ }^{1-16}$ Axons of the SPN exit through the ventral roots and synapse on postganglionic sympathetic neurons located within the spinal prevertebral ganglia. The postganglionic neurons then send their axons within the peripheral nerves and innervate the target organs, the heart and blood vessels (Figure 1).

The parasympathetic control of the cardiovascular system is conducted through the vagus nerve, which exits from the brain through the base of the skull and synapses with postganglionic neurons within the myocardium. ${ }^{17}$ There is no parasympathetic innervation of the peripheral vasculature.

SCI leads to disruption of the descending spinal cardiovascular pathways, resulting in sympathetic hypoactivity and unopposed prevalence of the intact vagal parasympathetic control. ${ }^{18}$ Sympathetic hypoactivity results in low resting blood pressure, loss of regular adaptability of blood pressure, and disturbed reflex control. ${ }^{6,8}$

\section{Abnormalities of cardiovascular control following SCI}

Blood pressure control is usually severely disrupted following SCI, although the manifestations of the altered blood pressure control tend to change with time following the injury. Soon after the injury (hours to days) there is a transient state of hypoexcitability of the isolated spinal cord, known as 'spinal shock', which is associated with flaccid paralysis of the muscles and absent tendon reflexes, in addition to impairment of spinal autonomic function. ${ }^{19}$ Coincident with spinal shock there is dilatation of the blood vessels, particularly those in the skin, which (combined with impaired autonomic reflexes) often results in profound hypotension, and is termed 'neurogenic shock'. ${ }^{20,21}$

Over time (days to weeks) the spinal shock resolves, but SCI patients are frequently troubled with sudden falls in blood pressure upon postural change, or following prolonged periods of sitting (orthostatic hypotension) characterised by dizziness, lightheadedness or even syncopal events. ${ }^{21,22}$ Conversely, SCI individuals can also be affected by sudden bouts of hypertension triggered by afferent stimuli below the level of the spinal 
Table 1 Causes of disrupted cardiovascular control following SCI

Loss of supraspinal (excitatory and inhibitory) control $^{26}$

Reduced sympathetic tone below the lesion ${ }^{6,27,28}$

Morphologic changes in sympathetic neurons ${ }^{29-31}$

Plasticity within spinal circuits ${ }^{31-33}$

Peripheral $\alpha$-adrenoceptor hyper-responsiveness ${ }^{34}$

cord lesion (autonomic dysreflexia) presenting symptomatically with pounding headache and upper body flushing. ${ }^{8}$ Previously, it was felt that the hypotensive episodes were confined to the acute period following SCI, and autonomic dysreflexia to the chronic phase of SCI. ${ }^{6}$ However, it has also been shown that autonomic dysreflexia can present in the early phases of SCI, ${ }^{23,24}$ and that orthostatic hypotension can persist for years, and often becomes worse with time. ${ }^{25}$

Numerous investigators identified various possible mechanisms that are likely to be responsible for the abnormal sympathetic cardiovascular control following SCI (Table 1). Firstly, there is a loss of descending supraspinal sympathetic excitatory and inhibitory control. ${ }^{6,26}$ The extent to which this disrupts sympathetic control is directly related to the level at which the SCI occurred. ${ }^{6,8,18}$ Secondly, there is a generalised reduction in sympathetic activity associated with low plasma adrenaline and noradrenaline levels. , $^{67,28}$ This is particularly pronounced in high-level (cervical and high thoracic) complete SCI. Thirdly, there are morphologic alterations that occur within SPN following SCI ${ }^{29,30}$ Initially after SCI there are signs of atrophy of SPN (possibly due to partial deafferentation from the loss of descending medullary input), which is followed by axonal sprouting and as a result, the possible formation of new and often inappropriate synaptic connections. ${ }^{30}$ Finally, it has also been identified that there is peripheral $\alpha$-adrenoreceptor hyper-responsiveness following SCI,${ }^{34}$ and this may play a significant role in the development of autonomic dysreflexia.

\section{Prevalence of orthostatic hypotension in SCI}

Orthostatic hypotension is defined by The Consensus Committee of the American Autonomic Society and the American Academy of Neurology (1996) as a decrease in systolic blood pressure of $20 \mathrm{mmHg}$ or more, or in diastolic blood pressure of $10 \mathrm{mmHg}$ or more, upon the assumption of an upright posture from a supine position, regardless of whether symptoms occur. ${ }^{35}$

There is a strong link between SCI and the presence of orthostatic hypotension. ${ }^{6,36-39}$ Orthostatic manoeuvres performed during physiotherapy and mobilisation are reported to induce blood pressure decreases diagnostic of orthostatic hypotension in $74 \%$ of SCI patients, and symptoms of orthostatic hypotension (such as lightheadedness or dizziness) in $59 \%$ of SCI individuals. ${ }^{38}$ This is likely to have a negative impact upon the ability of SCI individuals to participate in rehabilitation.
Orthostatic stress imposed using tilt testing (passive standing) is associated with a slightly lower incidence of orthostatic hypotension than physiotherapy manoeuvres. One study reports orthostatic hypotension during tilting in $57 \%$ of SCI patients, and symptoms associated with the hypotension in $25 \%$ of these patients. ${ }^{40}$ The reason why passive tilting is less likely to produce orthostatic hypotension is unclear, but the presence or absence of orthostatic hypotension is likely to be related, at least in part, to the type of orthostatic manoeuvre performed, and whether this is active or passive in nature. It is known, however, that the likelihood of experiencing orthostatic hypotension is greater in patients with higher spinal cord lesions, and thus it is more common in individuals with tetraplegia. ${ }^{6,36-38}$ These individuals also experience larger falls in blood pressure associated with postural change than those with paraplegia. ${ }^{38}$ There is also an increased risk of orthostatic hypotension in individuals who sustain a traumatic SCI than in nontraumatic injury such as spinal stenosis. ${ }^{39}$ Finally, it is notable that there are few reported incidences of orthostatic hypotension associated with SCI in the elderly, ${ }^{40}$ which is surprising since among able-bodied elderly persons, orthostatic hypotension is a common and troublesome phenomena. ${ }^{41}$ The reasons why there is little evidence of orthostatic hypotension in elderly SCI patients are unknown and warrant further investigation. However, it is known that during the last decade there has been a demographic change, whereby there has been an increase in the age of SCI individuals, ${ }^{42,43}$ and this may be associated with an increased incidence of orthostatic hypotension in SCI individuals. Clinicians working with elderly individuals with SCI should be aware of the combined impact of both age- and SCI-induced alterations in orthostatic cardiovascular control.

The extent to which SCI subjects are prone to orthostatic hypotension can be seen in Figure 2. This shows the changes in blood pressure and heart rate following passive movement from a supine to seated position in a subject with SCI (C5 ASIA B), in comparison to a healthy control volunteer. ${ }^{44}$ The severity of SCI was assessed using the ASIA/IMSOP assessment of motor and sensory impairment. ${ }^{45}$ Upon the assumption of a passive seated position, the SCI subject exhibited a marked, progressive decrease in blood pressures and relative postural tachycardia. In contrast, in the control subject, blood pressures were increased following the change in posture with little change in heart rate, due to the presence of normal descending cardiovascular control to spinal autonomic circuits.

\section{Mechanisms underlying orthostatic hypotension in SCI}

The precise mechanisms responsible for orthostatic hypotension in SCI patients are uncertain, and likely to be multifactorial. Factors likely to underlie orthostatic hypotension and symptoms of orthostatic 


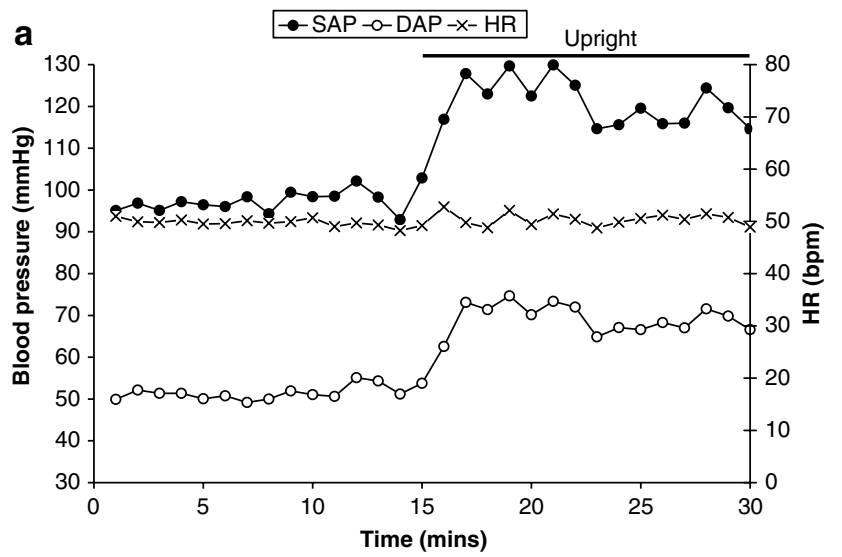

Table 2 Physiological factors that predispose to orthostatic hypotension in spinal cord-injured individuals

Predisposing factors

Sympathetic nervous system dysfunction ${ }^{6,28}$

Altered baroreceptor sensitivity ${ }^{62,64}$

Lack of skeletal muscle pumps ${ }^{49-51}$

Cardiovascular deconditioning ${ }^{69}$

Altered salt and water balance ${ }^{74}$

The level and severity of spinal cord injury, and the extent to which other systems are affected will play a significant role in the development of orthostatic hypotension

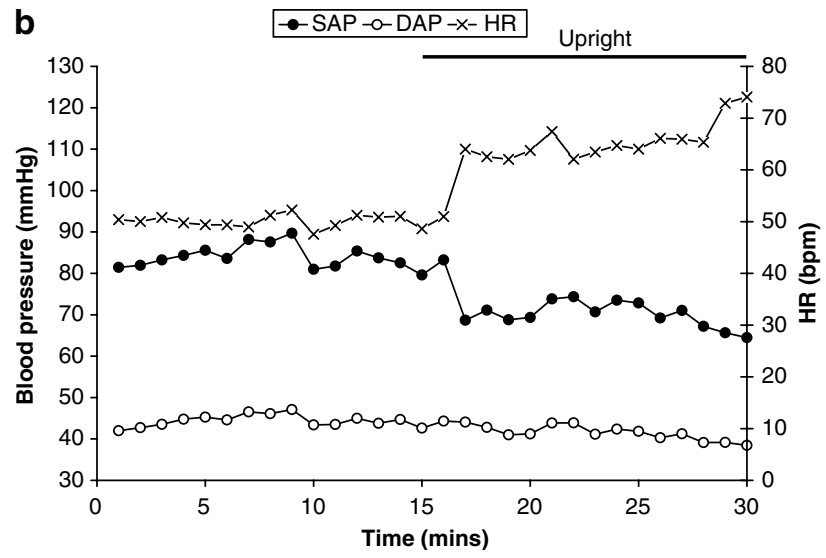

Figure 2 Blood pressure and heart rate responses to orthostatic stress in a healthy male control subject (a) and a man with chronic cervical SCI (C5 ASIA B; b) ${ }^{44}$ The SCI subject had complete destruction of descending autonomic pathways as assessed by the absence of sympathetic skin responses. Resting systolic (SAP) and diastolic (DAP) arterial pressures were higher in the control than the SCI subject. Resting heart rates (HR) were similar. Following the assumption of an upright seated position (indicated by the solid black line), the control subject had a marked increase in SAP and DAP, with little change in HR. In contrast, the SCI subject had a marked, progressive decrease in both SAP and DAP in the upright posture, characteristic of orthostatic hypotension, associated with a postural tachycardia of approximately $25 \mathrm{bpm}$

intolerance in SCI patients are discussed below, and summarised in Table 2.

\section{Sympathetic nervous system dysfunction}

As mentioned previously, the sympathetic nervous system plays a critical role in cardiovascular control. ${ }^{17}$ Parasympathetic control is usually preserved in $\mathrm{SCI},{ }^{8}$ but the synergistic relationship between parasympathetic and sympathetic control is lost, particularly in those with cervical and high thoracic SCI. Lesions above T6 disrupt supraspinal control to the splanchnic bed (and thus the major capacitance vessels) predisposing to orthostatic instability. The higher the level of injury, the

greater the level of impairment of efferent sympathetic nerves. ${ }^{6}$ This disruption of spinal sympathetic pathways would be likely to affect the vascular resistance responses to orthostasis, particularly in the dependent regions. In the upright position, there is normally a baroreceptor-mediated vasoconstriction, via an increase in tonic sympathetic outflow, in order to maintain blood pressure and cerebral perfusion. It has been demonstrated previously that these vascular resistance responses play a crucial role in cardiovascular control during orthostatic stress, ${ }^{46-48}$ and any impairment in vascular resistance responses following SCI will predispose to orthostatic intolerance. ${ }^{46,47}$

In the light of the alterations mentioned above, it is surprising that blood pressure control during orthostatic stress is not more severely impaired following SCI. One reason for this may be the fact that although resting supine sympathetic activity (and thus blood pressure) tends to be low after SCI, some studies do report an appropriate increase in peripheral resistance when upright in SCI individuals, despite presumed disruption of the sympathetic control. ${ }^{49-51}$ It is likely that some other mechanism is responsible for the increased peripheral resistance following orthostasis in SCI individuals. This could be related to production of catecholamines by the adrenal medulla, ${ }^{50}$ although recent evidence suggests that this is unlikely (see below), a large and rapid release of vasopressin upon postural change to levels sufficient to exert a pressor effect, ${ }^{52}$ spinal reflexes and veno-arteriolar reflexes ${ }^{6}$ or peripheral $\alpha$-adrenoceptor hyper-responsiveness. ${ }^{34}$ Although circulating supine resting catecholamine levels, particularly noradrenaline, are low in high SCI, there is a coincident hyper-responsiveness of $\alpha$-adrenoceptors below the lesion site ${ }^{34}$ such that if there were to be any increase in noradrenaline levels (eg via activation of the renin-aldosterone-angiotensin system) ${ }^{6}$ the peripheral $\alpha$-adrenoceptors would be likely to respond strongly. However, several investigations have shown that in SCI individuals, the levels of both adrenaline and noradrenaline are abnormally low while supine, ${ }^{6}$ and that SCI individuals fail to increase catecholamine levels in response to an orthostatic challenge (Claydon et al, unpublished observations; Figure 3). These observations are supported by earlier observations in a small number 
a

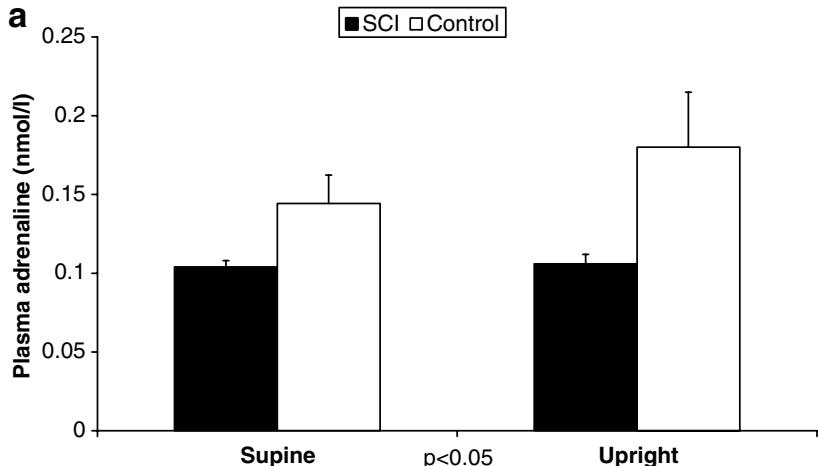

b
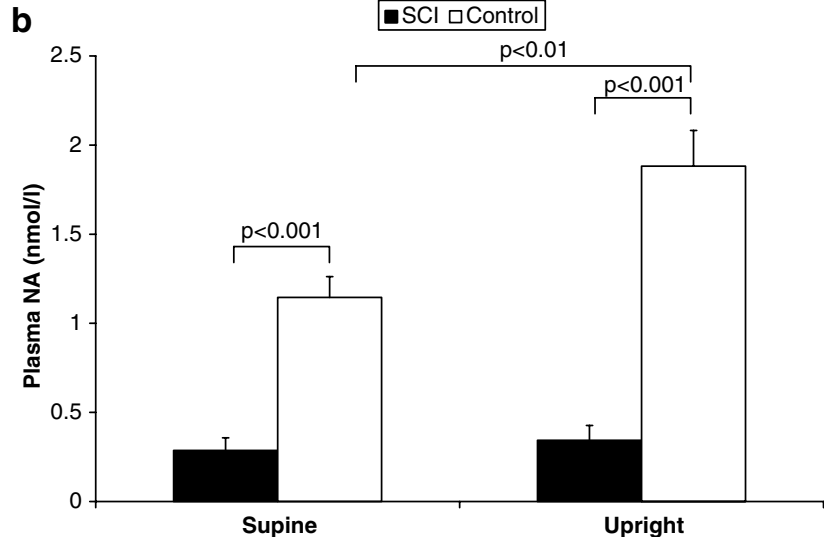

Figure 3 Plasma catecholamines in response to an orthostatic challenge in control volunteers $(n=7)$ and cervical SCI subjects $(n=7)$. Plasma adrenaline (a) and noradrenaline $(\mathrm{NA} ; \mathrm{b})$ are shown during supine rest and $5 \mathrm{~min}$ after the passive assumption of an upright seated position $\left(90^{\circ}\right)$. Supine adrenaline levels were similar in SCI and healthy controls. Movement to an upright position led to a small (although insignificant) rise in plasma adrenaline in controls, which was not seen in SCI subjects. NA levels were significantly lower in SCI during both the supine and upright condition. Following the assumption of an upright position, NA levels were markedly increased in controls, with no change in SCI subjects. This increase in the level of NA is due to tonic activation of autonomic circuits responsible for orthostatic stability in controls. However, in SCI individuals this descending control is markedly disrupted

of cervical SCI individuals in whom catecholamine levels failed to increase following head-upright tilting. ${ }^{53,54}$ These data suggest that there is severe impairment of descending tonic cardiovascular control to the spinal autonomic circuits in individuals with cervical SCI, such that there is insufficient sympathetic outflow to release noradrenaline, and consequently impaired vascoconstriction.

Another possible explanation for the ability to generate a small vascular resistance response in some SCI individuals may be related to partial preservation of descending tonic sympathetic supraspinal control to spinal circuits. ${ }^{18}$ Unfortunately, most studies examining cardiovascular dysfunction following SCI have used the traditional motor and sensory classification of the ASIA IMSOP assessment ${ }^{45}$ to evaluate severity of injury, which does not consider the integrity of autonomic pathways. Indeed, it has been shown recently that severe disruption of descending cardiovascular pathways was correlated with greater abnormalities of cardiovascular control in individuals with SCI. ${ }^{18}$ Furthermore, the importance of the assessment of autonomic control following SCI was highlighted in a recent review article. ${ }^{55}$

\section{Altered baroreflex function}

Baroreceptors are stretch receptors located in the aortic arch, carotid sinus and coronary arteries that respond to perturbations in arterial pressure and reflexly modulate sympathetic and parasympathetic outflow in order to maintain blood pressure homeostasis. Dysfunctional baroreceptor reflex control may present with various clinical conditions, depending on the nature of the disorder. This could, at least in part, be responsible for the low resting supine blood pressure in SCI individuals compared to able-bodied persons, particularly in patients with high-level (cervical and high thoracic) injuries. ${ }^{6}$ In addition, the circadian rhythms of blood pressure control, including circulating plasma noradrenaline and cortisol, are abolished in individuals with tetraplegia, and thus high-level lesions. ${ }^{56-58}$ This further highlights the baroreflex dysfunction in high-level SCI. ${ }^{59-61}$ In support of this notion, baroreflex function (assessed using spectral analysis of oscillations in heart period and blood pressure) during orthostatic stress was found to be abnormal in SCI patients with lesions at T3 or above. ${ }^{62}$ Furthermore, individuals with tetraplegia are also reported to have impaired baroreceptor responses to discrete stimulation of the carotid sinus using neck suction or neck pressure, whereby both the baroreceptor sensitivity and range of operation were reduced. ${ }^{63}$ However, the impairment in baroreceptor control may not be restricted to those individuals with high-level lesions. Additional research, again utilising spectral analyses, suggests that paraplegic patients also have altered baroreceptor responses to orthostasis, whereby the sympathetic response was blunted, and the response of vagal withdrawal was enhanced. ${ }^{64}$ These studies suggest an impairment of baroreceptor control in both high- and low-level lesions, which could contribute to orthostatic intolerance in SCI.

\section{Lack of skeletal muscle pumping activity}

The established test to assess the severity of orthostatic hypotension is to perform tilt testing. This involves passively moving the subject from a supine position into an upright position, with a vertical displacement of between 60 and $90^{\circ}$. One feature that is important during tilt testing is the passive nature of the test; the subject remains as still as possible and avoids movement of postural muscles. During head up tilt, the subject 
is supported either by a foot plate ${ }^{65}$ or a harness ${ }^{66}$ and is not tilted fully upright so that leg movements are avoided. During normal standing, on the other hand, postural muscles are continually reciprocally activated. This has the effect of compressing veins and pumping blood back to the heart, and is an important means of maintaining venous return when upright. ${ }^{67}$ SCI patients are lacking this skeletal muscle pumping effect, and this would predispose to circulatory hypokinesis by reducing venous return in the upright position and thus lowering stroke volume, cardiac output and blood pressure. ${ }^{50}$ The lack of skeletal muscle contractions during postural challenge has been demonstrated to have a powerful effect upon orthostatic changes in blood pressure in a number of studies. ${ }^{49-51}$ These investigators performed electrically stimulated leg muscle contractions in paraplegic and tetraplegic volunteers, simulating the skeletal muscle pumping effect normally present in able-bodied persons during standing. Electrically stimulated leg muscle contractions increased peripheral resistance, heart rate and cardiac output, and blunted the fall in stroke volume that normally occurs during orthostasis. ${ }^{49-51}$ However, perhaps most importantly, these induced contractions prevented or reduced the orthostatic hypotension normally seen in these SCI patients. Thus, it seems possible that the orthostatic hypotension associated with SCI is due, in part, to the failure to activate skeletal muscle pumps in paralysed dependant limbs during postural challenge.

\section{Cardiovascular deconditioning}

Cardiovascular deconditioning refers to a spectrum of physiological disorders that develop following prolonged bed rest, or exposure to microgravity (such as that which occurs during space flight). Following SCI, cardiovascular deconditioning is to be expected, due to the long periods of bed rest that are an inevitable consequence of the acute phase of the injury. Cardiovascular deconditioning is manifest by profound orthostatic intolerance, thought to be mediated via a diminished blood volume, decreased muscle or tissue pressure in the extremities (secondary to decreased muscle mass), or to functional alterations in the sympathetic nervous system. ${ }^{68}$ It has also been noted that microgravity-adapted animals have marked elevation of nitric oxide production in the kidney, heart, brain and systemic arteries, coupled with decreased nitric oxide production in the cerebral arteries. ${ }^{69}$ Nitric oxide acts on vascular smooth muscle to produce vasodilation, and is also known as endothelium-derived relaxing factor. ${ }^{70}$ Thus, the changes in nitric oxide noted would lead to enhanced peripheral vasodilatation (and reduced blood pressure) with a tendency for cerebral vasoconstriction (and hypoperfusion). These factors combined would worsen orthostatic hypotension. Thus, it has been suggested that cardiovascular deconditioning following SCI (due to prolonged bed rest or disruption of the efferent sympathetic nervous control) may be mediated, at least in part, by altered nitric oxide metabolism. ${ }^{69}$
The orthostatic intolerance that occurs coincident with cardiovascular deconditioning is characterised by postural hypotension and postural tachycardia. ${ }^{68}$ Cardiovascular deconditioning related to immobilisation is probably a major factor in the acute period of SCI. However, in individuals with chronic SCI it is less likely to be responsible for their orthostatic hypotension, as with time following injury individuals are mobilised, and any deconditioning effects are likely to be resolved.

\section{Altered salt and water balance}

In able-bodied individuals, orthostatic tolerance and postural blood pressure control are known to be greatly influenced by plasma volume, ${ }^{66}$ whereby larger plasma and/or blood volumes are associated with better tolerance to orthostasis. ${ }^{6,71}$ Similarly, expansion of plasma volume following salt supplementation improves orthostatic tolerance. $^{72,73}$ Thus, it would be expected that salt depletion and/or small plasma volumes would predispose to orthostatic hypotension. ${ }^{66}$ In patients with chronic SCI, there is evidence of impaired water and sodium retention in the seated position, and limited salt and water intake. ${ }^{25,74}$ Hyponatremia is also reported in acute SCI. ${ }^{75}$ Thus, hyponatremia, probably secondary to supraphysiological ADH activity in SCI ${ }^{51}$ is common among acute and chronic SCI patients. ${ }^{25,75,76}$ The combination of increased ADH secretion, low sodium intake, high sodium excretion and the resulting hyponatremia are likely to predispose towards small plasma volumes in SCI patients, and this, in turn, would exacerbate episodes of orthostatic hypotension in these individuals.

\section{Orthostatic hypotension and symptoms of cerebral hypoperfusion in SCI}

Orthostatic hypotension frequently results in cerebral hypoperfusion and symptoms of orthostatic intolerance such as dizziness or fainting (syncope). However, some individuals are relatively insensitive to hypotension and are able to maintain normal consciousness in the face of low arterial pressures. This is particularly evident in individuals suffering from autonomic failure, such as multiple system atrophy and pure autonomic failure. ${ }^{21,77-79}$ The mechanisms underlying this tolerance to hypotension are unclear, but since the ultimate cause of symptoms in these individuals is known to be due to cerebral hypoperfusion, it is likely that there is a shift in cerebral autoregulation in these individuals, whereby cerebral blood flow is maintained despite low perfusion pressures. ${ }^{77-79}$ Patients with SCI who demonstrate postural hypotension also seem to have altered cerebral haemodynamics, since many of them are able to tolerate profound hypotension and remain asymptomatic, and indeed, symptoms were only reported during documented orthostatic hypotension in approximately $60 \%$ of cases. ${ }^{38}$ 


\section{Cerebral autoregulation}

In able-bodied individuals, cerebral blood flow shows marked autoregulation, whereby pressure changes are buffered by changes in cerebrovascular resistance such that flow remains constant. ${ }^{80}$ When supine, blood is distributed evenly throughout the body and a mean arterial pressure of $85 \mathrm{mmHg}$, as measured at heart level, will also correspond to a mean cerebral perfusion pressure of $85 \mathrm{mmHg}$. However, when upright, cerebral arterial pressure is $15-30 \mathrm{mmHg}$ lower than that in the aortic arch due to the vertical height difference between the head and heart. ${ }^{80}$ This relative reduction in cerebral perfusion pressure provides a challenge to cerebral autoregulation, whereby cerebrovascular resistance must decrease in order to maintain constant flow (according to Ohms Law). Since a reduction in cerebral perfusion is the ultimate cause of presyncopal symptoms associated with orthostatic hypotension, an understanding of the control of cerebral autoregulation in SCI is crucial.

There is evidence that cerebral autoregulation is altered in SCI patients. In SCI patients with tetraplegia, a decline in blood pressure during tilting was associated with symptoms only in those individuals in whom cerebral blood flow was reduced to low levels. ${ }^{81}$ Indeed, in this study, cerebral blood flow was significantly lower in individuals with symptomatic orthostatic hypotension $(32.5 \pm 5 \mathrm{~cm} / \mathrm{s})$ compared to asymptomatic individuals $(40.9 \pm 8 \mathrm{~cm} / \mathrm{s}, P<0.02)$, even though the decrease in blood pressure with postural change was similar in the two groups (Figure 4). These investigators suggested, therefore, that autoregulation of cerebral blood flow, rather than systemic blood pressure, plays the dominant role in the apparent adaptation to orthostatic hypotension in SCI patients. However, it is remarkable that such low pressures (approximately $85 / 60 \mathrm{mmHg})^{81}$ should be tolerated. If the systemic blood pressure decreases to low levels, the cerebral perfusion pressure (in the upright position) will be reduced considerably further, due to the hydrostatic effect of the height difference between the head and heart when upright. The crucial factor in determining consciousness, however, is not specifically cerebral blood flow, but rather cerebral oxygenation. ${ }^{82}$ SCI patients, although demonstrating a much larger fall in blood pressure upon orthostasis than able-bodied controls, show a similar reduction in cerebral oxygenation when upright. ${ }^{83}$ Thus, although the systemic circulation is compromised in SCI patients, cerebral oxygenation is maintained as well as in able-bodied controls. In support of this, another study by the same group found that both able-bodied controls and SCI individuals had similar reductions in cerebral oxygenation to simulated standing using supine lower body suction, despite the SCI subjects having larger decreases in systemic blood pressure. ${ }^{82}$ Similarly, they also noted that the SCI group had larger falls in cerebral blood flow during the orthostatic stress. The greater decline in cerebral blood flow seems contrary to the other studies, and the control of cerebral blood flow in SCI requires further examination. It may be that the

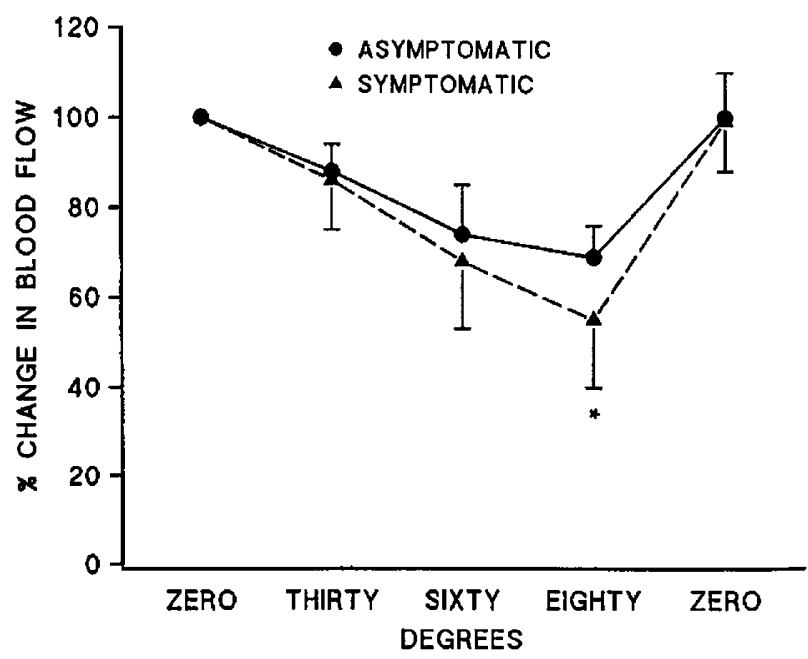

Figure 4 Responses of middle cerebral artery blood flow velocity to the upright position in SCI individuals with symptomatic and asymptomatic orthostatic hypotension. Cerebral blood flow was recorded in 10 asymptomatic and nine symptomatic SCI individuals with orthostatic hypotension induced by passive tilting to 30,60 and $80^{\circ}$, and is expressed as the percentage change from the baseline supine levels. Cerebral blood flow decreased in both groups with tilting $(P<0.01)$. However, the decrease in blood flow was significantly greater in the symptomatic individuals (*denotes $P<0.02$ ). Cerebral blood flow returned to baseline levels upon resumption of the supine position. Supine blood pressures and the reduction in blood pressures during tilting were similar in both groups. Reprinted with permission from Paraplegia (Gonzalez F, Chang JY, Banovac K, Messina D, MartinezArizala A, Kelley RE. Autoregulation of cerebral blood flow in patients with orthostatic hypotension after spinal cord injury. Paraplegia 1991; 29: 1-7); copyright (1991) Macmillan Publishers Ltd; http://www.nature.com

extent to which the cerebral blood flow is controlled is related to the level, or completeness of the SCI, a factor that was not controlled for in these studies. Furthermore, we have to acknowledge that at the present time there is no data available regarding the correlation between the severity of injury to autonomic pathways following SCI and cerebral haemodynamics.

\section{Common carotid artery haemodynamics}

Much of the blood supply to the cerebral circulation is provided via the carotid arteries, and in addition to supplying blood to the head they also contain the carotid baroreceptors. ${ }^{17}$ This is important because during orthostasis the carotid baroreceptors lie above heart level, and as such are activated by the relative fall in pressure due to the hydrostatic effects of gravity, ${ }^{80}$ and normally bring about the coordinated reflex responses to orthostasis. In SCI, the baroreceptor pathways may be impaired, ${ }^{64}$ and in addition there may, ${ }^{82}$ or may not, ${ }^{81,83}$ be a disruption of cerebral blood flow during standing.

For these reasons, carotid artery haemodynamics could provide additional information concerning cere- 
brovascular control in SCI patients. It has been shown that common carotid artery (CCA) diameter and flow, during supine and when tilted upright, were similar in SCI patients with paraplegia and able-bodied controls. ${ }^{84}$ However, SCI patients with tetraplegia had significantly greater CCA diameter and flow in the supine position. In all three groups there was a reduction in CCA diameter and flow upon tilting, but in tetraplegic patients, this change was greater, such that the tilted levels became similar to those in paraplegic patients and controls. The authors propose that individuals with tetraplegia may show an adaptation in CCA haemodynamics that is beneficial in terms of orthostatic tolerance, which maintains flow both to the carotid baroreceptive regions, and towards the brain, in the face of greater falls in blood pressure during standing than paraplegic patients or able-bodied controls.

\section{Implications of impaired blood pressure control in SCI}

It is well-known that orthostatic hypotension affects the ability of individuals with SCI to participate in activities likely to provoke falls in blood pressure. This has a marked impact upon activities of daily living, particularly participation in rehabilitation programmes, and severely affects quality of life. ${ }^{38}$ However, in addition to these profound episodes of orthostatic hypotension, it is likely that the low resting blood pressures that are common in SCI (particularly in cervical SCI) would also have a deleterious affect upon the health of SCI individuals. Indeed, it is known that even moderate resting hypotension (systolic pressure below $110 \mathrm{mmHg}$ ) is associated with deficits in cognitive performance. ${ }^{85}$ Furthermore, orthostatic hypotension is commonly associated with fatigue, ${ }^{86,87}$ which is an other factor that adversely impacts upon quality of life and participation in rehabilitation. In addition, it is possible that those individuals with more severe orthostatic hypotension, and thus presumably the greatest impairment of cardiovascular control, may also be more prone to other cardiovascular abnormalities such as episodes of autonomic dysreflexia. The long-term risks of acute swings in blood pressure from very low levels associated with orthostatic hypotension to very high levels associated with autonomic dysreflexia are not known. However, it is well recognised that the initiation and progress of atherosclerosis may be related to repeated bouts of hypertension, leading to shear stress and consequent endothelial damage. ${ }^{88,89}$ This would provide a mechanism linking the blood pressure dysregulation of SCI individuals and their increased cardiovascular mortality. ${ }^{10}$

\section{Management of orthostatic hypotension in SCI}

The management of orthostatic hypotension in individuals with SCI is essentially similar to the treatment of autonomic failure in the able-bodied population. Although the aetiology of the autonomic impairment in autonomic failure is different to that of SCI, the subsequent presentation of orthostatic hypotension (and other autonomic dysfunctions) may be similar in the two disorders, and thus the management of orthostatic hypotension in both SCI and the autonomic failure syndromes is similar. The treatment of orthostatic hypotension in relation to other disorders has been extensively reviewed elsewhere, ${ }^{87,90,91}$ and a full discussion of the treatment paradigms is beyond the scope of this review.

In brief, nonpharmacologic measures should form the first line of therapy. Individuals should be advised regarding the mechanisms underlying orthostatic hypotension, and common factors likely to provoke an episode. Further advice should be given on the maintenance of plasma volume by ensuring an adequate salt and fluid intake, ${ }^{47,70}$ and the avoidance of diuretics such as alcohol and caffeine. SCI individuals should be educated as to the symptoms of orthostatic hypotension and encouraged to assume a recumbent or semirecumbent position should they occur. The use of compression bandages or support stockings to restrict venous pooling in the splanchnic region and dependent limbs should be encouraged. ${ }^{87,90,91}$ Patients should also be advised to avoid vasodilatory stresses such as heat stress or common vasodilators, including alcohol, and to take regular small meals to minimize postprandial hypotension. ${ }^{87,90,91}$ It has also been shown that sleeping with the bed head raised by $10-20^{\circ}$ increases plasma volume and orthostatic tolerance in able-bodied individuals, ${ }^{80,92}$ and this should be encouraged in individuals with SCI and orthostatic hypotension. If these measures are ineffective, pharmacological therapy may be appropriate. This most commonly involves the expansion of plasma volume with fludrocortisone, ${ }^{92,93}$ or increasing peripheral vasoconstriction with the $\alpha$-adrenergic agonist midodrine. $^{94,95}$ A summary of these management options is shown in Table 3.

\section{Summary}

Orthostatic hypotension is a distressing and troublesome disorder, which is frequently associated with SCI. The mechanisms underlying orthostatic hypotension

Table 3 Strategies for the management of orthostatic hypotension in individuals with SCI

\begin{tabular}{l} 
Nonpharmacological management \\
Advice and avoidance of precipitating factors ${ }^{87,90,91}$ \\
Increased salt and fluid intake ${ }^{48,72}$ \\
Abdominal compression bandages and/or support \\
stockings ${ }^{87,90,91}$ \\
Sleeping with the bed head raised by $10-20^{\circ 80,92}$ \\
Minimize postprandial hypotension \\
Pharmacotherapy \\
Fludrocortisone \\
Midodrine \\
\hline Nonpharmacological measures should form the first line of \\
therapy
\end{tabular}

Advice and avoidance of precipitating factors $87,90,91$

Increased salt and fluid intake ${ }^{48,72}$

Abdominal compression bandages and/or support stockings ${ }^{87,90,91}$

Sleeping with the bed head raised by $10-20^{\circ 80,92}$

Pharmacotherapy

Fludrocortisone ${ }^{92,93}$

Midodrine ${ }^{94,95}$

therapy 
following SCI are unclear, but are likely to be multifactorial. Some possible contributing factors are lack of tonic sympathetic control (particularly in those with high level lesions), impaired baroreceptor regulation, lack of skeletal muscle pumping activity in the dependant limbs of paralysed individuals, cardiovascular deconditioning following prolonged periods of bed rest and hyponatremia with the resultant hypovolemia. It does seem, however, that some protective adaptations occur in SCI patients that serve to limit the symptoms of orthostatic hypotension. These involve mainly adaptations in the cerebral circulation, sufficient to help to maintain cerebral oxygenation in the face of falling perfusion pressures. Greater emphasis should be placed upon the evaluation and management of autonomic function in patients following SCI.

\section{References}

1 Ackery A, Tator C, Krassioukov A. A global perspective on spinal cord injury epidemiology. J Neurotrauma 2004; 21: $1355-1370$.

2 Calaresu FR, Yardley CP. Medullary basal sympathetic tone. Ann Rev Physiol 1988; 50: 511-524.

3 Osborn JW, Taylor RF, Schramm LP. Determinants of arterial pressure after chronic spinal transection in rats. Am J Physiol 1989; 256: R666-R673.

4 Krassioukov AV, Weaver LC. Episodic hypertension due to autonomic dysreflexia in acute and chronic spinal cordinjured rats. Am J Physiol 1995; 268: H2077-H2083.

5 Maiorov DN, Fehlings MG, Krassioukov AV. Relationship between severity of spinal cord injury and abnormalities in neurogenic cardiovascular control in conscious rats. J Neurotrauma 1998; 15: 365-374.

6 Mathias CJ, Frankel HL. Autonomic disturbances in spinal cord lesions. In: Mathias CJ, Bannister R (eds). Autonomic Failure: A Textbook of Clinical Disorders of the Autonomic Nervous System, 4th edn. Oxford University Press: Oxford 2002, pp 494-513.

7 Osborn JW, Taylor RF, Schramm LP. Chronic cervical spinal cord injury and autonomic hyperreflexia in rats. Am J Physiol 1990; 8: R169-R174.

8 Teasell RW, Arnold MO, Krassioukov A, Delaney G. Cardiovascular consequences of loss of supraspinal control of the sympathetic nervous system after spinal cord injury. Arch Phys Med Rehab 2000; 81: 506-516.

9 Krum $\mathrm{H}$ et al. Risk factors for cardiovascular disease in chronic spinal cord injury patients. Paraplegia 1992; 30: 381-388.

10 Devivo MJ, Krause JS, Lammertse DP. Recent trends in mortality and causes of death among persons with spinal cord injury. Arch Phys Med Rehabil 1999; 80: 1411-1419.

11 Garshick E et al. A prospective assessment of mortality in chronic spinal cord injury. Spinal Cord 2005; 43: 408-416.

12 Verberne AJ, Owens NC. Cortical modulation of the cardiovascular system. Prog Neurobiol 1998; 54: 149-168.

13 Krassioukov AV, Weaver LC. Anatomy of the autonomic nervous system. Phys Med Rehabil 1996; 10: 1-14.

14 Krassioukov AV, Fehlings MG. Effect of graded spinal cord compression on cardiovascular neurons in the rostroventrolateral medulla. Neuroscience 1999; 88: 959-973.
15 Lebedev VP, Krasyukov AV, Nikitin SA. Electrophysiological study of sympathoexcitatory structures of the bulbar ventrolateral surface as related to vasomotor regulation. Neurosci 1986; 17: 189-203.

16 Reis DJ, Morrison S, Ruggierdo DA. The C1 area of the brainstem in tonic and reflex control of blood pressure. Hypertension 1988; 11: I8-I13.

17 Shepherd RFJ, Shepherd JT. Central nervous control of the cardiovascular system. In: Bannister R, Mathias CJ (eds). Autonomic Failure: A Textbook of Clinical Disorders of the Autonomic Nervous System, 4th edn. Oxford University Press: Oxford 2002, pp 54-93.

18 Furlan JC, Fehlings MG, Shannon P, Norenberg MD, Krassioukov AV. Descending vasomotor pathways in humans: correlation between axonal preservation and cardiovascular dysfunction after spinal cord injury. $J$ Neurotrauma 2003; 20: 1351-1363.

19 Ditunno JF, Little JW, Tessler A, Burns AS. Spinal shock revisited: a four-phase model. Spinal Cord 2004; 42: 383-395.

20 Atkinson PP, Atkinson JLD. Spinal shock. Mayo Clin Proc 1996; 71: 384-389.

21 Mathias CJ, Mallipeddi R, Bleasdale-Barr K. Symptoms associated with orthostatic hypotension in pure autonomic failure and multiple system atrophy. J Neurol 1999; 246: 893-898.

22 Mathias CJ, Frankel HL. Clinical manifestations of malfunctioning sympathetic mechanisms in tetraplegia. J Auton Nerv Syst 1983; 7: 303-312.

23 Krassioukov AV, Furlan JC, Fehlings MG. Autonomic dysreflexia in acute spinal cord injury: an under-recognised clinical entity. $J$ Neurotrauma 2003; 20: 707-715.

24 Silver JR. Early autonomic dysreflexia. Spinal Cord 2000; 38: $229-233$.

25 Frisbie JH, Steele DJR. Postural hypotension and abnormalities of salt and water metabolism in myelopathy patients. Spinal Cord 1997; 35: 303-307.

26 Claus-Walker J, Halstead LS. Metabolic and endocrine changes in spinal cord injury: II (section 1). Consequences of partial decentralization of autonomic nervous system. Arch Phys Med Rehab 1982; 63: 569-575.

27 Maiorov DN, Weaver LC, Krassioukov AV. Relationship between sympathetic activity and arterial pressure in conscious spinal rats. Am J Physiol 1997; 272: H625-H631.

28 Wallin BG, Stiernberg L. Sympathetic activity in man after spinal cord injury. Brain 1984; 107: 183-198.

29 Krassioukov AV, Bunge RP, Puckett WR, Bygrave MA. The changes in human spinal cord sympathetic preganglionic neurons after spinal cord injury. Spinal Cord 1999; 37: 6-13.

30 Krassioukov AV, Weaver LC. Morphological changes in sympathetic preganglionic neurons after spinal cord injury in rats. Neuroscience 1996; 70: 211-225.

31 Krassioukov AV, Johns DG, Schramm LP. Sensitivity of sympathetically correlated spinal interneurons, renal sympathetic nerve activity, and arterial pressure to somatic and visceral stimuli after chronic spinal injury. $J$ Neurotrauma 2002; 19: 1521-1529.

32 Krenz NR, Weaver LC. Sprouting of primary afferent fibers after spinal cord transection in the rat. Neuroscience 1998; 85: 443-458.

33 Krenz NR, Meakin SO, Krassioukov AV, Weaver LC. Neuralizing intraspinal nerve growth factor blocks autonomic dysreflexia caused by spinal cord injury. $J$ Neuroscience 1999; 19: 7405-7414. 
34 Arnold JM, Feng QP, Delaney GA, Teasell RW. Autonomic dysreflexia in tetraplegic patients: evidence for alpha-adrenoceptor hyper-responsiveness. Clin Auton Res 1995; 5: 267-270.

35 The Consensus Committee of the American Autonomic Society and the American Academy of Neurology. Consensus statement on the definition of orthostatic hypotension, pure autonomic failure, and multiple system atrophy. Neurology 1996; 46: 1470.

36 Lopes P, Figoni S. Current literature on orthostatic hypotension and training in SCI patients. Am Correct Ther 1982; 36: 893-898.

37 Blackmer J. Orthostatic hypotension in spinal cord injured patients. J Spinal Cord Med 1997; 20: 212-217.

38 Illman A, Stiller K, Williams M. The prevalence of orthostatic hypotension during physiotherapy treatment in patients with an acute spinal cord injury. Spinal Cord 2000; 38: 741-747.

39 McKinley WO, Tewksbury MA, Godbout CJ. Comparison of medical complication following non-traumatic and traumatic spinal cord injury. J Spinal Cord Med 2002; 25: 88-93.

40 Cariga P, Ahmed S, Mathias CJ, Gardner BP. The prevalence and association of neck (coat hanger) pain and orthostatic (postural) hypotension in human spinal cord injury. Spinal Cord 2002; 40: 77-82.

41 Kenny RA. Syncope in the elderly: diagnosis, evaluation and treatment. J Cardiovasc Electrophysiol 2003; 14: S74-S77.

42 Krassioukov AV, Furlan JC, Fehlings MG. Medical co-morbidities, secondary complications and mortality in elderly with acute spinal cord injury. J Neurotrauma 2003; 20: 391-399.

43 Calancie B, Molano MR, Broton JG. Epidemiology and demography of acute spinal cord injury in a large urban setting. J Spinal Cord Med 2005; 28: 92-96.

44 Krassioukov AV, Elliott S, Claydon VE. Cardiovascular responses to a 'sit up test' in men with cervical spinal cord injury. Clin Auton Res 2005; 15: 334.

45 Maynard FM et al. International standards for neurological and functional classification of spinal cord injury. Spinal Cord 1997; 35: 266-274.

46 Brown CM, Hainsworth R. Forearm vascular responses during orthostatic stress in control subjects and patients with posturally related syncope. Clin Auton Res 2000; 10: 57-61.

47 Bush VE, Wight VL, Brown CM, Hainsworth R. Vascular responses to orthostatic stress in patients with postural tachycardia syndrome (POTS), in patients with low orthostatic tolerance, and in asymptomatic controls. Clin Auton Res 2000; 10: 279-284.

48 Claydon VE, Hainsworth R. Salt supplementation improves orthostatic cerebral and peripheral vascular control in patients with syncope. Hypertension 2004; 43: 1-5.

49 Raymond J, Davis GM, Bryant G, Clarke J. Cardiovascular responses to an orthostatic challenge and electricalstimulation-induced leg muscle contractions in individuals with paraplegia. Eur J Appl Physiol Occup Physiol 1999; 80: 205-212.

50 Faghri PD, Yount JP, Pesce WJ, Seetharama S, Vootto JJ. Circulatory hypokinesis and functional electric stimulation during standing in persons with spinal cord injury. Arch Phys Med Rehabil 2001; 82: 1587-1595.

51 Faghri PD, Yount J. Electrically induced and voluntary activation of physiologic muscle pump: a comparison between spinal cord-injured and able-bodied individuals. Clin Rehab 2002; 16: 878-885.

52 Wall BM et al. Characteristics of vasopressin release during controlled reduction in arterial pressure. J Lab Clin Med 1994; 124: 554-563.

53 Mathias CJ, Christensen NJ, Corbett JL, Frankel HL, Goodwin TJ, Peart WS. Plasma catecholamines, plasma renin activity and plasma aldosterone in tetraplegic man, horizontal and tilted. Clin Sci Mol Med 1975; 49: 291-299.

54 Mathias CJ, Christensen NJ, Frankel HL, Peart WS. Renin release during head-up tilt occurs independently of sympathetic nervous activity in tetraplegic man. Clin Sci 1980; 59: 251-256.

55 Ellaway PH et al. Towards improved clinical and physiological assessments of recovery in spinal cord injury: a clinical initiative. Spinal Cord 2004; 42: 325-337.

56 Krum H, Louis WJ, Brown DJ, Jackman GP, Howes LG. Diurnal blood pressure variation in quadraplegic chronic spinal cord injury patients. Clin Sci 1991; 80: 271-276.

57 Mayorov DN, Adams MA, Krassioukov AV. Telemetric blood pressure monitoring in conscious rats before and after compression injury of the spinal cord. $J$ Neurotrauma 2001; 18: 727-736.

58 Munakata M, Kameyama J, Kanazawa M, Nunokawa T, Moriai N, Yoshinaga K. Circadian blood pressure rhythm in patients with higher and lower spinal cord injury: simultaneous evaluation of autonomic nervous activity and physical activity. J Hypertens 1997; 15: 1745-1749.

59 Conway J, Boon N, Jones JV, Sleight P. Involvement of the baroreceptor reflexes in the changes in blood pressure with sleep and mental arousal. Hypertension 1983; 5: 746-748.

60 Nakazato T, Shikama T, Toma S, Nakajima Y, Masuda Y. Nocturnal variation in human sympathetic baroreflex sensitivity. J Auton Nerv Sys 1998; 70: 32-37.

61 Makino M, Hayashi H, Takezawa H, Hirai M, Saito H, Ebihara S. Circadian rhythms of cardiovascular functions are modulated by the baroreflex and the autonomic nervous sustem in the rat. Circulation 1997; 96: 1667-1674.

62 Munakata M, Kameyama J, Nunokawa T, Ito N, Yoshinaga K. Altered Mayer wave and baroreflex profiles in high spinal cord injury. Am J Hypertens 2001; 14: 141-148.

63 Convertino VA, Adams WC, Shea JD, Thompson CA, Hoffler GW. Impairment of carotid-cardiac vagal baroreflex in wheelchair-dependent quadriplegics. Am J Physiol 1991; 260: R576-R580.

64 Wecht JM, de Meersman RE, Weir JP, Spungen AM, Bauman WA. Cardiac autonomic responses to progressive head-up tilt in individuals with paraplegia. Clin Auton Res 2004; 13: 433-438.

65 El-Bedawi KM, Hainsworth R. Combined head up tilt and lower body suction - a test of orthostatic tolerance. Clin Auton Res 1994; 4: 41-47.

66 El-Sayed H, Hainsworth R. Relationship between plasma volume, carotid baroreceptor sensitivity and orthostatic tolerance. Clin Sci 1995; 88: 463-470.

67 Guyton AC, Richardson TQ, Langston JB. Regulation of cardiac output and venous return. Clin Anesth 1964; 3: $1-34$.

68 Graveline DE. Cardiovascular deconditioning: role of blood volume and sympathetic neurohormones. Life Sci Space Res 1964; 2: 287-298.

69 Vaziri ND. Nitric oxide in microgravity-induced orthostatic intolerance: relevance to spinal cord injury. $J$ Spinal Cord Med 2003; 26: 5-11. 
70 Ignarro LJ, Buga GM, Wood KS, Byrns RE, Chaudhuri G. Endothelium-derived relaxing factor produced and released from artery and vein is nitric oxide. Proc Natl Acad Sci USA 1987; 84: 9265-9269.

71 Claydon VE et al. Orthostatic tolerance and blood volumes in Andean high altitude dwellers. Exp Physiol 2004; 89: 565-571.

72 Mtinangi BL, Hainsworth R. Early effects of oral salt on plasma volume, orthostatic tolerance and baroreceptor sensitivity in patients with syncope. Clin Auton Res 1998; 8: 231-235.

73 El-Sayed H, Hainsworth R. Salt supplement increases plasma volume and orthostatic tolerance in patients with unexplained syncope. Heart 1996; 75: 134-140.

74 Frisbie JH. Postural hypotension, hyponatremia, and salt and water intake: case reports. J Spinal Cord Med 2004; 27: 133-137.

75 Karlsson AK, Krassioukov AV. Hyponatremia-induced transient visual disturbances in acute spinal cord injury. Spinal Cord 2004; 42: 204-207.

76 Soni BM, Vaidyanthan S, Watt JWH, Krishman KR. A retrospective study of hyponatremia in tetraplegic paraplegic patients with a review of the literature. Paraplegia 1994; 32: 597-607.

77 Bondar RL et al. Cerebrovascular and cardiovascular responses to graded tilt in patients with autonomic failure. Stroke 1997; 28: 1677-1685.

78 Horowitz DR, Kaufmann H. Autoregulatory cerebral vasodilatation occurs during orthostatic hypotension in patients with primary autonomic failure. Clin Auton Res 2001; 11: 363-367.

79 Hetsel A, Reinhard M, Guschlbauer B, Braune S. Challenging cerebral autoregulation in patients with preganglionic autonomic failure. Clin Auton Res 2003; 13: $27-35$.

80 Hainsworth R. Syncope and fainting. In: Mathias CJ, Bannister R (eds). Autonomic Failure: A Textbook of Clinical Disorders of the Autonomic Nervous System, 4th edn. Oxford University Press: Oxford 2002, pp 761-781.

81 Gonzalez F, Chang JY, Banovac K, Messina D, MartinezArizala A, Kelley RE. Autoregulation of cerebral blood flow in patients with orthostatic hypotension after spinal cord injury. Paraplegia 1991; 29: 1-7.

82 Houtman S, Serrador JM, Colier JM, Strijbos DW, Shoemaker K, Hopman MTE. Changes in cerebral oxygenation and blood flow during LBNP in spinal cordinjured individuals. J Appl Physiol 2001; 91: 2199-2204.

83 Houtman S, Colier WN, Oeseburg B, Hopman MT. Systemic circulation and cerebral oxygenation during head-up tilt in spinal cord injured individuals. Spinal Cord 2000; 38: 158-163.

84 Wecht JM, Radulovic M, Lessey J, Spungen AM, Bauman WA. Common carotid and common femoral arterial dynamics during head-up tilt in persons with spinal cord injury. J Rehab Res Dev 2004; 41: 89-94.

85 Duschek S, Weisz N, Schandry R. Reduced cognitive performance and prolonged reaction time accompany moderate hypotension. Clin Auton Res 2003; 13: 427-432.

86 Wessely S, Nickson J, Cox B. Symptoms of low blood pressure: a population study. $B M J$ 1990; 301: 362-365.

87 Freeman R. Treatment of orthostatic hypotension. Semin Neurol 2003; 23: 435-442.

88 Boyd GW. Stress and disease: the missing link. A vasospastic theory. II The nature of degenerative arterial disease. Med Hypotheses 1978; 4: 420-431.

89 Johansson BB. Hypertension mechanisms causing stroke. Clin Exp Pharm Physiol 1999; 26: 563-565.

90 Bannister R, Mathias CJ. The management of postural hypotension. In: Mathias CJ, Bannister R (eds). Autonomic Failure: A Textbook of Clinical Disorders of the Autonomic Nervous System, 4th edn. Oxford University Press: Oxford 2002, pp 622-645.

91 Mathias CJ. Autonomic diseases: management. J Neurol Sureosurg Psychiatry 2003; 74: iii42-iii47.

92 Ten Harkel AD, Van Lieshout JJ, Wieling W. Treatment of orthostatic hypotension with sleeping in the head-up tilt position, alone and in combination with fludrocortisone. J Intern Med 1992; 232: 139-145.

93 Groomes TE, Huang CT. Orthostatic hypotension after spinal cord injury: treatment with fludrocortisone and ergotamine. Arch Phys Med Rehabil 1991; 72: 56-58.

94 Mukand J, Karlin L, Barrs K, Lublin P. Midodrine for the management of orthostatic hypotension in patients with spinal cord injury: a case report. Arch Phys Med Rehabil 2001; 82: 694-696.

95 Barber DB, Rogers SJ, Fredrickson MD, Able AC. Midorine hydrochloride and the treatment of orthostatic hypotension in tetraplegia: two cases and a review of the literature. Spinal Cord 2000; 38: 109-111. 